\title{
A Simplified Electronic Voting Machine System
}

\author{
Md. Murshadul Hoque \\ Department of Applied Physics, Electronics and Communication Engineering \\ University of Chittagong, Chittagong, Bangladesh \\ m.hoque@cu.ac.bd
}

\begin{abstract}
In this paper, an Electronic Voting Machine (EVM) system is proposed which is in operation as transparent as the digital system. The Simplified Electronic Voting Machine (SEVM) responds on some flow of pulses coming from the switch operated by voter and produces the output of the counting values i.e. total casting votes of individual nominee and displays it.The machine is controlled both automatically and manually to operate the system for successive voters and to ensure that a voter can give only one vote to his/her chosen candidate of the same position. The manual controlling system must be operated by presiding officer who have the authorization to check and to declare a voter valid after checking some unique information e.g. NID number where as the automatic controlling happens whenever a voter pushs a switch to vote. Designing and implementing of this SEVM system is very plain and convenient due to having discrete digital circuitry.
\end{abstract}

Keywords: Electronic Voting Machine, Discrete Digital Circuitry, Counting, Authorization, Transparency

\section{Introduction}

Electronic Voting Machine is one of the most important topics to urgue about for the political parties actually from last consecutive few years. In the traditional system [1-3], the election commission has to print individual ballot-paper for each and every voter. A voter uses "seal and ink" to vote their chosen candidate. And sometimes, however, many votes become invalid for giving the "seal" in unexpected region. Again, the piracy in voting [1, 3-6] and lack of transparency in counting [1,3-6] are the major shortcomings of the traditional system. This process of counting votes is also very time consuming. In some countries, people are practising electronic machines [3] for voting. These systems are designed with sophisticated electronic elecments and devices such as microcontroller, comparator, complexed multiplexer, memory etc ICs which are much more costly, inflexible, inavailable and complex [3]. It always needs expart technical handlings for maintainance that makes all the people imprudent of it and has the probability of technical larceny of programming [7-10].

In order to come out from the complexity of paper-seal-ink or chic EVM, I wanted to make a simple machine. The basic feature of my proposed machine which is simple to construct and use, flexible and cost effective. It will also confirm security, transparency and fast response [11-13]. 
SEVM consists of four main units (Figure 1) such as voting unit, controlling unit, counting unit and display unit. Here, in voting unit the pulse is generated by voter which is controlled by controller unit in both manually and automatically to pass to the counting unit and finally, it is counted and displayed. Voter is just to press a switch to make a vote and the SEVM counts and displays the total votes sequentially for each and every candidate separately. The SEVM is so simple that it is handy in size and easily portable.

The rest of this paper is organized as follows. In Section 2, I introduce the design overview of our proposed system. In Section 3, the procedure of hardwire design and circuit operation is illustrated. The performance analysis is presented in Section 4, and finally I conclude this paper in conclusion section.

\section{Design Overview}

In order to make the idea real, I reley on "pulses". I use "pulses" as the effect of a vote and count those to make the result. Some very elementary logic gates, counter and innocent switches $[14,15]$ are used to design a simple system in its constractional and operational as well where no programmable memory element is used. The whole overview of the proposed design is dipicted by the Figure 1.

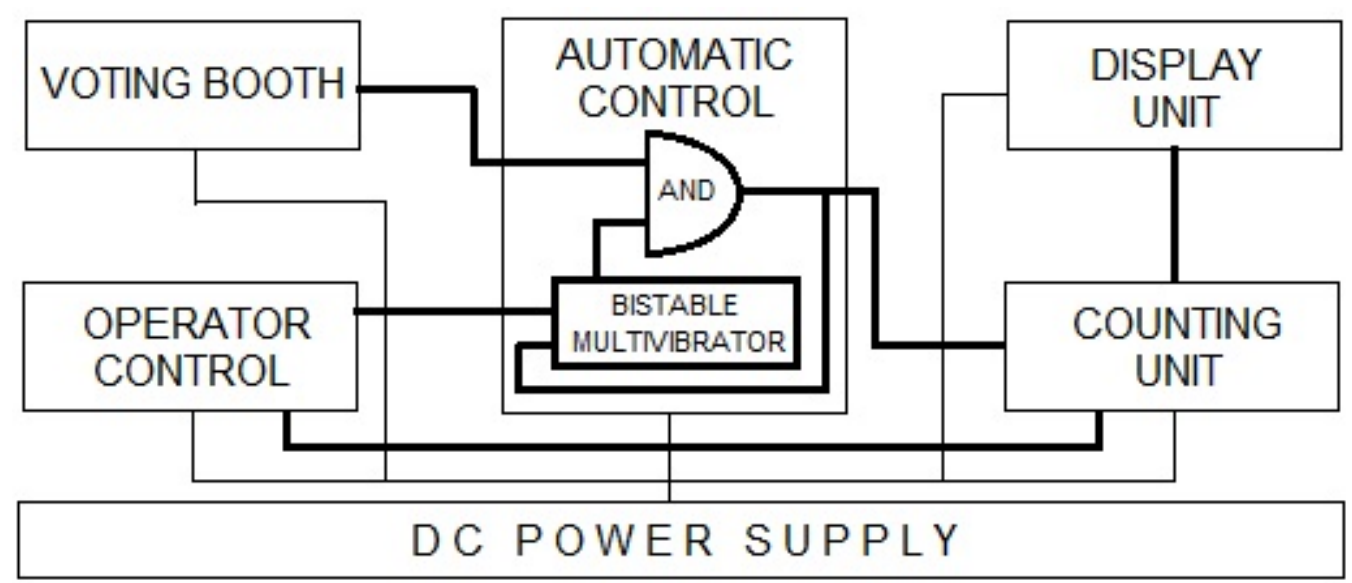

Figure 1. Block Diagram of SEVM

The pulse, that is generated by a voter by pressing a switch which is marked with the name of a candidate in the booth, goes to drive the corresponding counter and display units. The operator-control unit controls to allow the voter to vote, to transmit the pulse to automatic control unit and to enable the counting and display units. In this system, a voter can vote simply by pressing a switch which consumes roughly 3-4 seconds where it is very time consuming and clumsy for a voter to collect and to seal a ballot, and to fold it as instructed in the existing traditional systems [1-3]. Here, a voter cannot give more than one vote. He or she can press the switch as many times as he or she wants. But the system is made in such a way that, it will count only one vote for single voter. That ensures the security and the transparency of polling. More detailed of these mechanism will be described in the operation and performance sections.

The design is incorporated with a separate section of counting and display units for an individual candidate. The exclusive property of this machine is that it counts every vote just within a few miliseconds. So, the polling officers need not to wait for completion of total voting process, like the treditional system. 


\section{Hardware Design and Circuit Operation}

SEVM responses the pulses sent by the voters, and counts the number of pulses corresponding to single candidate and displays it independently. In the circuit, shown in Figure 2, two switches are incorporated for two candidates named as " $\mathrm{X}$ " and ' $\mathrm{Y}$ ' for a position.

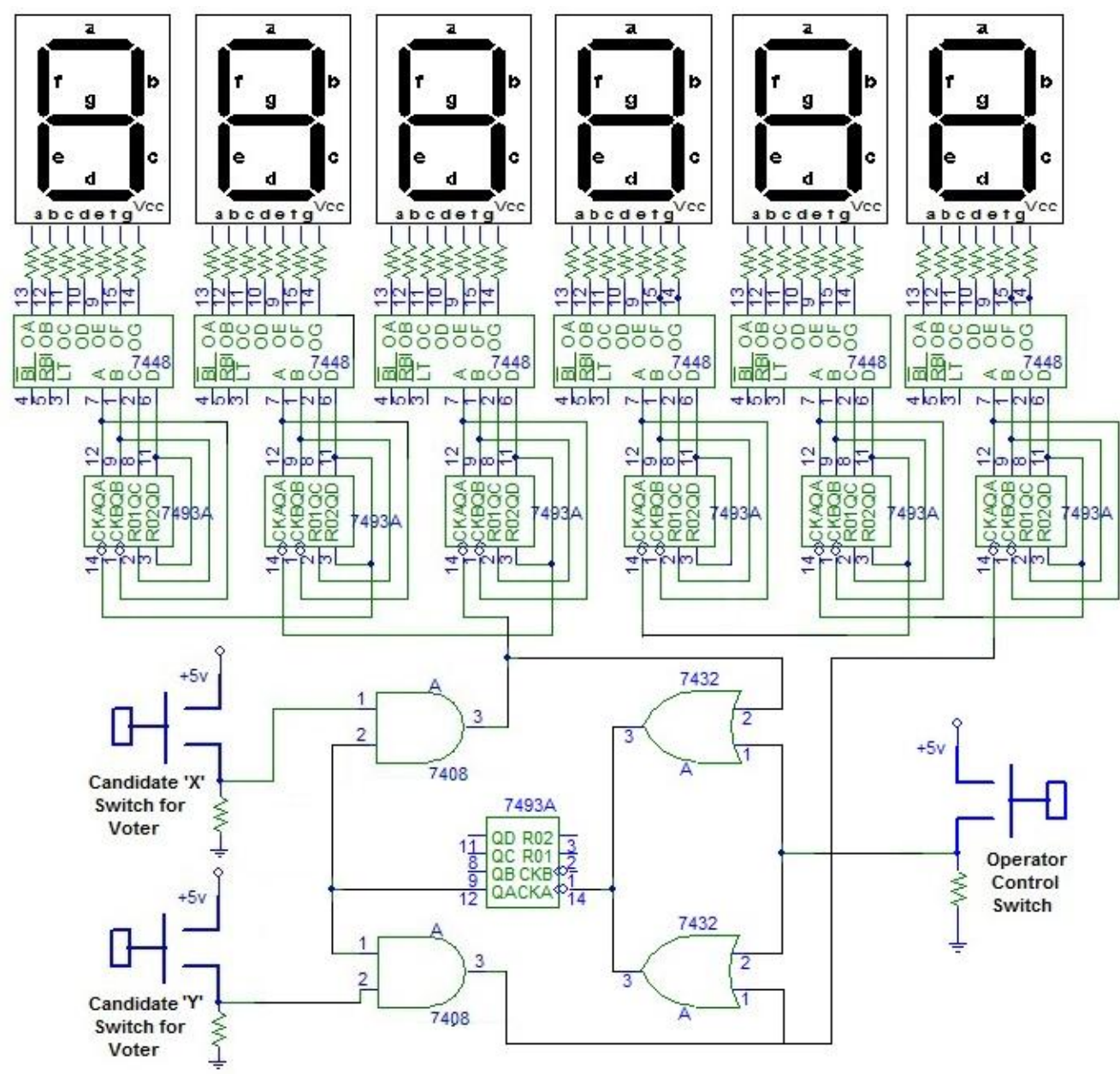

Figure 2. Circuit Diagram of SEVM for a position of two Candidates

Firstly, operator will permit the voters to provide their valuable votes by checking the NID cards and pictures with the information available in the polling office. Then the operator allows the voter by pressing his control switch to count his/her vote that comes from the respective candidate's switch. Here, control pulse triggers the bistable multivibrator switch [15] to high stage and lets the machine ready to accept the pulse from the candidate's switch. Whenever a voter presses any switch, the pulse is processed to count by BCD counter 7493IC [14] at the counter section, and the output stage of bistable switch becomes low simultaneously that in turn makes the machine disable to allow pulses again for any illegal presses in the booth section until the operator permits another voter to vote. That will happen in the same procedure. Finally, the count value is displayed by 7-segment display with decoder [14] at the display section for the both candidates individually. The SEVM is decorated by adapting some additional switches and LEDs for setting and resetting the 
machine, and for assuring the system and for pressing the switch by voter OK respectively. Here, it must be mentioned clearly for the vast application of this machine that more candidates for a position and other section for other position can be incorporated by inserting similar units as more as necessary.

\section{Performance Analysis}

The SEVM is presented, in Figure 2, for only two candidates who are competing for the same position. And it can also be designed for any number of candidates for several positions. The operation of the designed circuit is successcully examined and simulated by PSPICE [16] and DSCH [17] softwares. The timing diagram of the simulation is illustrated in the Figure 3.

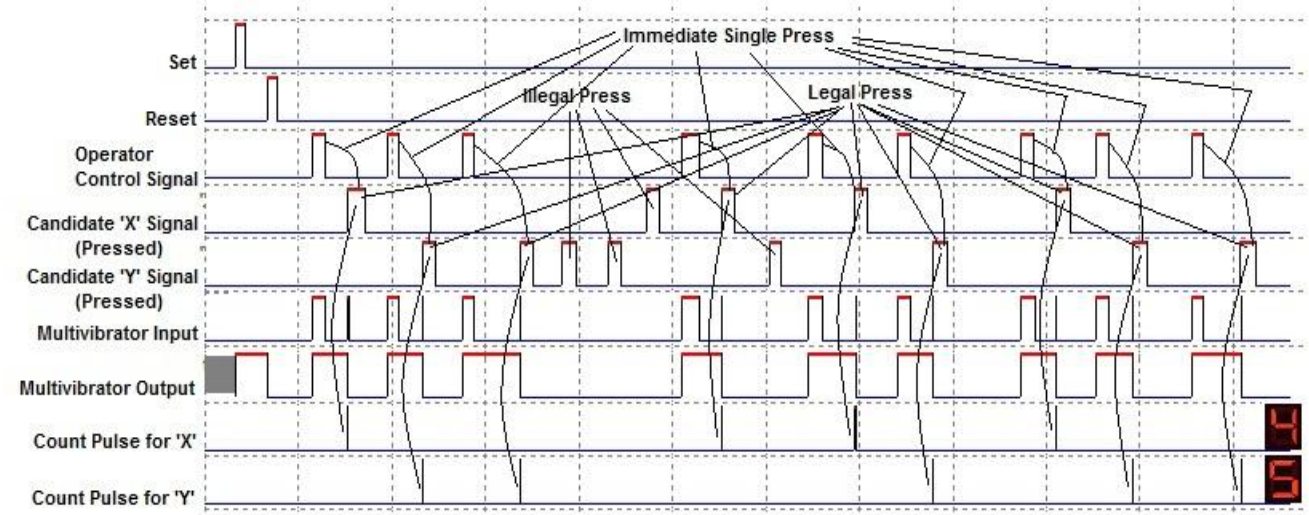

Figure 3. Timing Diagram of SEVM Operation

The signals including Set, Reset, Operator control signal, Candidates' signals, Multivibrator input and output, and Count pulses for different candidates are waved in this figure. Here it is seen that when the operator generates a pulse by pushing the switch, just immediate pulse i.e. "Immediate Single Press" from the switch of a candidate is considered as "Legal Press" to count but the others are rejected as "Illegal Press". The multivibrator input signal shows the series of pair pulses of the control pulse and the candidate count pulse to sustain the output of multivibrator HIGH. At last, the count value for each candidate is displayed.

\section{Discussion}

The process of election with transparency and security is imperative in any country to ensure the right of voting for the citizen. The proprietary SEVM system is more advantageous comparing with the traditional and other electronic systems in the points of cost, flexibility, portability and effortless understanding, use and maintenance. As an example, to increase the capacity 10 times, it will cost only few dollars but in the existing system it would demand printing of many ballot papers with lot of dollers. Moreover, since all of the components are electronic, this system needs significantly low power. And the whole operation of the machine is logical and transparent due to the use of electronic and non-programmable components in this design. It is also compatible to interface with the computer system $[9,10,18]$, further to analyse the vote casting and the whole polling systems through out the divisions as well as country by internetworking system with enhanced security $[19,20]$. 


\section{References}

[1] S. P. Everett, M. D. Byrne and K. K. Greene, "Measuring the usability of paper ballots: Efficiency, effectiveness, and satisfaction", Proceedings of the Human Factors and Ergonomics Society 50th Annual Meeting, (2006) October 16-20; Santa Monica, USA.

[2] S. P. Everett, K. K. Greene, M. D. Byrne, D. S. Wallach, K. Derr, D. Sandler and T. Torous, "Electronic Voting Machines versus Traditional Methods: Improved Preference, Similar Performance", CHI Proceedings: Measuring, Business, and Voting, (2008) April 5-10; Florence, Italy.

[3] M. Patil, V. Pimplodkar, A. R. Zade, V. Vibhute and R. Ghadge, "A Survey on Voting System Techniques", International Journal of Advanced Research in Computer Science and Software Engineering, vol. 3, no. 1, (2013).

[4] B. Harris, "Black Box Voting: Vote Tampering in the 21st Century", Elon House/Plan Nine, (2003) July.

[5] D. W. Jones, "Problems with Voting Systems and the Applicable Standards", Testimony before the U.S. House of Representatives' Committee on Science, (2001) May.

[6] Diebold Election Systems Inc., "Checks and balances in elections equipment and procedures prevent alleged fraud scenarios", http://www.diebold.com/checksandbalances.pdf, (2003) July.

[7] J. Kelsey, "Strategies for software attacks on voting machines", NIST Workshop on Threats to Voting Systems, (2005) September; NIST.

[8] T. Kohno, A. Stubblefield, A. D. Rubin and D. S. Wallach, "Analysis of an electronic voting system", IEEE Symposium on Security and Privacy, (2004) May; California, USA.

[9] M. I. Shamos, "Computerized Voting-Evaluating the Threat", Proc. Third ACM Conf. on Computers, Freedom \& Privacy, (1993) March; San Francisco, USA.

[10] P. G. Neumann, "Risks in Computerized Elections", Inside Risks 5, CACM 33, (1990) November 11, pp. 170.

[11] A. J. Feldman, J. A. Halderman and E. W. Felten, "Security Analysis of the Diebold AccuVote-TS Voting Machine”, USENIX/ACCURATE Electronic Voting Technology Workshop (EVT'07), (2007) August; Boston, USA.

[12] D. Jefferson, "A Security Analysis of the Secure Electronic Voting and Registration System (SERVE)", (2004) January, http://www.servesecurityreport.org/paper.pdf.

[13] J. Bannet, "Hack-a-Vote: Security Issues with Electronic Voting Systems", IEEE Security and Privacy Magazine, (2004) January/February.

[14] R. J. Tocci and N. S. Widmer, "Digital System Principles and Applications", Pearson/Prentice Hall, (2004).

[15] S. L. Gupta and V. Kumar, "A Handbook of Electronics", Pragati Prakashan, India (2008).

[16] Berkley Spice, SPICE8, Electrical and Electronic Simulation Software, (1970).

[17] L. Theremin, DSCH 2, Digital Schematic Editor and Simulator, (1987).

[18] A. Keller, A. Dechert, K. Auerbach, D. Mertz, A. Pearl and J. Hall, "A pc-based open-source voting machine with an accessible voter-verifiable paper ballot", Open Voting Consortium, (2004).

[19] California Internet Voting Task Force, "A Report on the Feasibility of Internet Voting", (2000) January, http://www.ss.ca.gov/executive/ivote/.

[20] A. D. Rubin, "Security considerations for remote electronic voting", Communications of the ACM, vol. 45, no. $12, \mathbf{( 2 0 0 2 )}$.

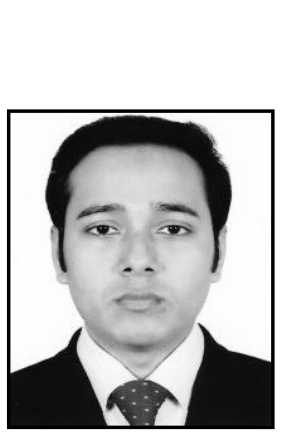

\section{Author}

\section{Md. Murshadul Hoque}

He received B.Sc. (4-year) degree in Electrical and Electronic Engineering from Chittagong University of Engineering and Technology (CUET), Bangladesh in 2006. Currently, he is working as an Assistant Professor in the Department of Applied Physics, Electronics and Communication Engineering at University of Chittagong, Bangladesh. His research interests, within power electronics, include integrated design, power system control \& automation, power conversion, alternative energy sources \& technologies and smart power grid. 
International Journal of Advanced Science and Technology Vol.62, (2014) 